\title{
Acute Changes on Pulmonary Pressure Following Percutaneous Secundum Atrial Septal Defect Closure
}

\author{
Jessica Wiryanto ${ }^{1,{ }^{*}}$, Ingrid M. Pardede ${ }^{2}$, Sunanto $\mathrm{Ng}^{1,2}$ \\ ${ }^{1}$ Faculty of Medicine, University of Pelita Harapan, Jakarta, Indonesia, ${ }^{2}$ Department of Cardiology, Siloam Hospital Lippo \\ Village, Jakarta, Indonesia \\ *correspondence: jessicawiryanto@gmail.com
}

\begin{abstract}
Introduction: Pulmonary Hypertension (PH) can be found in 9 to $35 \%$ of patients with atrial septal defect (ASD). It is a consequence of increased left to right shunt resulting in volume overload of the right heart and pulmonary circulation. ${ }^{1}$ If left untreated pulmonary hypertension might further develop into right heart failure, arrhythmia, and thromboembolic events. ${ }^{2}$ Percutaneous closure has become a preferred treatment in patients with secundum type ASD. It does not only increases exercise tolerance, it also improves heart hemodynamics. ${ }^{5}$ This case evaluates the hemodynamic effects of percutaneous atrial septal defects closure on pulmonary hypertension.
\end{abstract}

Case presentation:

Case 1. Forty years old female came to our clinics with complaint of chest pain, palpitations and dyspnea on effort since last 1 year. On examinations, she had wide fixed splitting heard best at 3rd ICS. Chest X-ray showed cardiomegaly and peripheral pruning. ECG showed sinus rhythm with incomplete right bundle branch block. Transthoracic echocardiography revealed inferior vena cava dilatation (IVC) and IVC collapse of less than $50 \%$, right atrial and ventricular dilatation, no LV D-shaped. Tricuspid valve pressure gradient (TVG) was $37 \mathrm{mmHg}$ with estimated mean pulmonary arterial pressure (mPAP) of $36.77 \mathrm{mmHg}$ and estimated right systolic ventricular pressure of 57 $\mathrm{mmHg}$. Right heart catheterization confirms the presence of pulmonary hypertension with PA pressure $26 / 24 \mathrm{mmHg}$. Three months later evaluation with transesophageal echocardiography (TEE) was done, there was 1.6-2.3 cmsecundum ASD with left to right shunts and pulmonary hypertension with TVG 35mmHg. Rim assessment proved suitable for device closure. She underwent device closure procedure with amplatzer septal occluder (ASO). After the procedure, heart hemodynamics was re-evaluated with transthoracic echocardiography. Estimated mPAP was $36 \mathrm{mmHg}$, and TVG was $39 \mathrm{mmHg}$. On follow up, patient feel better with increased functional class.

Case 2. A thirty four years old female came to our clinics with a complaint of palpitation since 1 year ago and recurrent dyspnea for the past three months and exercise intolerance.On examinations, she had wide fixed splitting with ECG showing incomplete right bundle branch block. There was no history of paradoxical embolism. TEE was performed confirming diagnosis of secundum Atrial Septal Defects with left to right shunts and an estimated diameter of $1.6-2.3 \mathrm{~cm}$. TVG was $30 \mathrm{mmHg}$. As rim was suitable for ASD closure, she was then planned to undergo TEE guided ASD closure. After closure, assessment showed good placement of septal occluder device, with TVG $23 \mathrm{mmHg}$ and there was no IVC dilatation, IVC collapse was less than $50 \%$. On follow up there was no complication. On follow up patient no longer experience dyspnea and there was no other complaints.

Discussion: Closure of interatrial septal defects closes the left to right shunt, resulting in reduction of volume overload on the right heart and pulmonary circulation. Thus pulmonary vascular disease might be prevented or reversed with early correction of the defects. In this paper we evaluate changes in pulmonary hypertension by echocardiography. Estimation of PA pressure by echocardiography can be made by assessing estimating tricuspid valve pressure gradient. Aside from that, RA pressure estimation can be used to evaluate right heart hemodynamics. Estimation of RA pressure can be made with IVC diameter and degree of inspiratory collapse. The first case displayed no changes in TVG while having increased clinical performance. This could be caused by a long standing pulmonary hypertension leading to pulmonary vascular remodeling causing increased pulmonary vascular resistance and reduced compliance. Or because of the reduced compliance of the left ventricle in comparison to right ventricular compliance. Mean while, the second case displayed changes in TVG after device closure and clinical improvements. This difference in TVG evaluation result might be caused by a ten year age difference as studies have shown that aging correlates with increased pulmonary systolic arterial pressure. Despite the difference in $\mathrm{PH}$ changes after percutaneous ASD closure, both patients display clinical improvements. 